\title{
Trends in the incidence of human papillomavirus-related noncervical and cervical cancers in Alberta, Canada: a population-based study
}

\author{
Lorraine Shack PhD, Harold Y. Lau MD, Longlong Huang MSc, Corinne Doll MD, Desirée Hao MD
}

\section{Abstract}

Introduction: Recent epidemiologic studies have suggested that the incidence of noncervical cancers associated with human papillomavirus (HPV) is increasing. We assessed temporal, age-specific and sex-specific changes in the incidence of HPV-associated cancers in a population-based study.

Methods: We used the Alberta Cancer Registry, a registry of all cancers diagnosed in the province of Alberta, Canada, to identify patients with cancers of the oropharynx, cervix, vulva, vagina, anus and penis (cancers associated with HPV) between Jan. 1, 1975, and Dec. 31, 2009. We estimated the age-standardized incidence of each cancer by sex- and age-specific group and assessed the annual percentage change using joinpoint regression.

Results: The age-standardized incidence of oropharyngeal cancers increased for each 5-year interval of the study period among men (annual percentage change 3.4, $<$ 0.001) and women (annual percentage change 1.5, $p=0.009$ ). For anal cancers, the age-standardized rates increased among women (annual percentage change 2.2, $p<0.001$ ) and men (annual percentage change 1.8, $p=0.008$ ). The age-standardized incidence of cervical cancer increased with age, reaching an annual percentage change of -3.5 among women aged $75-84$ years $(p=0.04)$. The rates of other HPV-associated cancers (vulvar, vaginal and penile) showed little change.

Interpretation: Our findings showed increases in the incidence of the HPV-associated cancers of the oropharynx and anus among men and women, and increases in cervical cancer among younger women. The incidence of HPV-related cancers in younger age groups should continue to be monitored. Programs to prevent HPV infection, such as vaccination, should be considered for males as well as females.

\section{A}

ccording to world-wide estimates, human papillomavirus (HPV) infection is responsible for $5.2 \%$ of all cancers. ${ }^{1,2}$ The association between HPV and cervical cancer has been well-known for many decades, but the link between HPV and cancers of the anus, penis, vagina, vulva and oropharynx has only recently been confirmed. ${ }^{3}$ Cervical cancer rates have been declining in Canada and the United States, consistent with the successful adoption of Papanicolaou smear screening programs, ${ }^{4,5}$ but the incidence of other HPV-related cancers has been increasing. The increasing incidence of these HPV-related cancers has been attributed to changes in lifestyle-related risk factors, most notably sexual behaviour. ${ }^{5}$ Analysis of trends in incidence and birth cohorts in both the US and the United Kingdom have shown an increased risk of HPV-related cancer in more recent birth cohorts and recent time periods, $, 5,6$ and these increases have been attributed to generational changes in sexual patterns and increased exposure to $\mathrm{HPV}^{6}$
HPV vaccines are now broadly used in the prevention of cervical cancer. In Alberta, HPV vaccination programs were implemented in 2008 and, as in many other jurisdictions, have been funded only for females. Although HPV vaccination programs may reduce the incidence of HPV-related cancers other than cervical cancer, ${ }^{6}$ they remain the subject of much public policy debate in Canada.' In this study, we sought to examine trends in noncervical HPV-related cancers over time and compare them with relative changes in cervical cancer and non-HPV-related cancers with similar risk factors.

Competing interests: None declared.

This article has been peer reviewed.

Correspondence to: Lorraine Shack, Lorraine.shack@albertahealthservices.ca

CMAJ Open 2014.DOI:10.9778/cmajo.20140005 


\section{OPEN}

\section{Methods}

\section{Patient population}

We defined HPV-associated cancers as those for which there is evidence to support their association with $\mathrm{HPV}^{3}$ We identified cancers diagnosed between Jan. 1, 1975, and Dec. 31, 2009, from the Alberta Cancer Registry using the following International Classification of Diseases for Oncology, 3rd Revision (ICD-O-3) codes for the HPV-associated group: cancers of the anus (C20-C21), cervix (C53), oropharynx (C019, C024, C028, C090-C099, C102, C108, C140, C142 and C148), vulva (C51), vagina (C52) and penis (C60). The Alberta Cancer Registry is an accredited registry with population-based coverage since the 1950s. It currently covers a population of 3.6 million and has achieved the highest level for complete, accurate and timely incidence data as recognized by the North American Association of Central Cancer Registries. ${ }^{8}$ For a comparison of trends in incidence, we identified a group of non-HPV-associated, modifiable-risk cancers that were diagnosed over the same period from the Alberta Cancer Registry: cancers of the lip, oral cavity and pharynx (C00.0C0.69, C07.9-C10.9), lung (C33, C34), endometrium (C54), ovary (C56) and colon (C18, C19).

All tumours diagnosed since 2007 were coded according to the ICD-O-3; for those diagnosed in earlier years, we converted the codes to ICD-O-3 codes. Age at diagnosis was categorized by 10 -year age group $(<35,35-44,45-54,55-64$, $65-74,75-84)$, and year of diagnosis was assigned to a 5-year period (1975-1979, 1980-1984, 1985-1989, 1990-1994, 1995-1999, 2000-2004, 2005-2009). Because of the small number of cases of cancers of the anus, oropharynx and vagina among patients younger than 35 years, for these cancers, the youngest group was defined as age less than 45 years. Because of the small number of cancers of the lip, oral cavity and pharynx, trends for these were estimated over time for all ages. Patients aged 85 or older were excluded because of the small number of cases.

\section{Statistical analysis}

We calculated age-standardized and age-specific rates (per 100000 population with $95 \%$ confidence intervals [CT]) by sex and cancer type for each 5-year period of diagnosis from 1975 to 2009. Rates were age-standardized to the 1991 Canadian cancer population. We identified trends in age-standardized and age-specific rates over time, and changes in these trends, using joinpoint regression. Joinpoint uses a regression model to describe trends by a sequence of trend lines, or annual percentage change, and to identify points where any significant changes in trends occur. We modelled trends using the Monte Carlo permutation method to select the optimum number of points at which incidence trends changed (either in direction or magnitude). ${ }^{9}$ The maximum number of joinpoints was limited to 5 as recommended. ${ }^{9}$ To assess trends by age group where incidence was low, we analyzed 5-year periods, thereby estimating the percentage change between periods. Findings were described as significant if the $p$ value was less than 0.05 . Analyses were conducted with SAS software (version 9.1; SAS Institute Inc.) and the Joinpoint Regression Program (version 4.0.4; Statistical Methodology and Applications Branch, Surveillance Research Program, National Cancer Institute).

\section{Ethics approval}

This retrospective study was reviewed and approved by the Alberta Cancer Research Ethics Committee, Alberta Health Services.

\section{Resullts}

Between 1975 and 2009, 8120 HPV-associated cancers were diagnosed in Alberta. Most were cervical cancer (56.1\%) and oropharyngeal cancer (17.9\%) (Table 1). The HPV-related cancers were generally diagnosed at a younger age than the non-HPV-associated cancers (lung, endometrium, ovary and colon) (Table 2). Cervical cancer was diagnosed at the youngest age (mean 47.0 years, $95 \%$ CI $46.5-47.5$ ), followed by oropharyngeal (mean 60.1 years, $95 \%$ CI 59.4-60.6), ovarian (mean 60.3 years, $95 \%$ CI 58.5-61.4) and anal cancer (mean 62 years, 95\% CI 61.2-63.3).

Significant increases in the incidence of HPV-associated cancers of the anus, oropharynx and vulva, and of non-HPVassociated cancer of the lung among women, were noted between 1975 and 2009. The age-standardized incidence of anal cancer among women doubled between 1975 and 2009, from 0.7 (95\% CI $0.5-0.9)$ per 100000 to 1.5 (95\% CI 1.1 1.9) per 100000 ; the annual percentage change was 2.2 $(p<0.001)$ (Appendix 1e available at www.cmajopen.ca/content /2/3/E127/suppl/DC1). The increase in incidence per 5-year interval of anal cancers among women was highest among those less than 45 years (annual percentage change 5.3, $p=0.014$ ) and those $45-54$ years (annual percentage change $6.4, p=0.011$ ) and decreased with advancing age (Figure 1A). The age-standardized incidence of anal cancer among men increased from 0.5 (95\% CI 0.4-0.6) per 100000 in 1975 to 0.9 (95\% CI 0.8-1.0) per 100000 in 2009, with an annual percentage change of $1.8(p=0.008)$ (Appendix 1e). Among men, the incidence per 5-year interval increased in all age groups, with the annual percentage change ranging from 1.8 in the $45-54$ and 55-64 age groups to 3.8 among those less than 45 at diagnosis (Figure 1B).

For oropharyngeal cancer, the increase in incidence per 5year interval among men (annual percentage change 3.4, $p<0.001$ ) was double that among women (annual percentage change 1.5, $p=0.009$ ) between 1975 and 2009 (Appendix 1f). The age-standardized incidence of oropharyngeal cancer increased from 1.2 (95\% CI 1.1-1.3) to 3.7 (95\% CI 3.5-3.9) per 100000 among men, as compared with an increase from 0.6 (95\% CI $0.5-0.7$ ) to 1.0 (95\% CI 0.9-1.1) per 100000 among women. For women, the incidence increased with advancing age, from 0.01 (95\% CI 0.0-0.1) per 100000 among those less than 45 years to 3.3 (95\% CI 2.9-3.7) per 100000 among those aged 65-74 years and 2.6 (95\% CI 2.3-2.9) per 100000 among those aged 75-84. The same was true for the rise in incidence per 5-year interval (annual percentage change -1.7 among those $<45 \mathrm{yr}$ to 1.9 among those $75-85 \mathrm{yr}$; test for increase in age, 
$p=0.11$ ), although this was not significant (Figure 2A). The incidence per 5-year interval for oropharyngeal cancer among men decreased with advancing age (annual percentage change 6.9 among those $35-44$ yr to 1.5 among those $75-84 \mathrm{yr}$ ); this was also not statistically significant $(p=0.23)$ (Figure $2 \mathrm{~B}$ ).

There was little change in the incidence over time of HPVassociated cancers of the vulva, vagina and penis (Appendix 1a). The increase in age-standardized incidence of vulvar cancer was small (annual percentage change 0.07, $p=0.043$ ) (Appendix 1a). In comparison, the age-standardized incidence of cervical cancer declined over this period (annual percentage change $-1.1, p<0.001$ ) (Appendix 1g). The decline in cervi- cal cancer incidence increased with age, reaching an annual percentage change of -3.5 among women aged 75-84 (test for trend: $p<0.001$ ) (Figure 3).

In comparison, among non-HPV-related cancers, the agestandardized incidence of cancers of the lip and oral cavity was stable among men and women (Appendix 1h). The agestandardized incidence of lung cancer among men decreased after 1987 (annual percentage change $-1.5, p<0.001$ ), but among women, it continued to increase, from 13.4 (95\% CI 13.2-13.6) per 100000 in 1975 to 47.3 (95\% CI 46.6-48.0) per 100000 in 2009 (Appendix 1b). There was little change in the age-standardized incidence of endometrial cancer since 1980

\begin{tabular}{|c|c|c|c|c|c|c|}
\hline \multirow[b]{2}{*}{ Characteristic } & \multicolumn{6}{|c|}{ HPV-related cancers; no. (\%) of patients } \\
\hline & $\begin{array}{c}\text { Anus } \\
n=721\end{array}$ & $\begin{array}{c}\text { Cervix } \\
n=4557\end{array}$ & $\begin{array}{c}\text { Oropharynx } \\
n=1454\end{array}$ & $\begin{array}{c}\text { Vulva } \\
n=793\end{array}$ & $\begin{array}{l}\text { Vagina } \\
n=266\end{array}$ & $\begin{array}{c}\text { Penis } \\
n=329\end{array}$ \\
\hline \multicolumn{7}{|l|}{ Sex } \\
\hline Male & $264(36.6)$ & - & $1098(75.5)$ & - & - & $329(100.0)$ \\
\hline Female & $457(63.4)$ & $4557(100.0)$ & $356(24.5)$ & $793(100.0)$ & $266(100.0)$ & - \\
\hline \multicolumn{7}{|l|}{ Age, yr } \\
\hline$<35$ & \multirow[t]{2}{*}{$80(11.1)^{*}$} & $1123(24.6)$ & \multirow[t]{2}{*}{$107(7.4)^{*}$} & $29(3.7)$ & \multirow[t]{2}{*}{$27(10.1)^{*}$} & $5(1.5)$ \\
\hline $35-44$ & & $1294(28.4)$ & & $99(12.5)$ & & $27(8.2)$ \\
\hline $45-54$ & $145(20.1)$ & $830(18.2)$ & $393(27.0)$ & $118(14.9)$ & $43 \quad(16.2)$ & $49(14.9)$ \\
\hline $55-64$ & $179(24.8)$ & $549(12.1)$ & $445(30.6)$ & $110(13.9)$ & 61 (22.9) & $79(24.0)$ \\
\hline $65-74$ & $153(21.2)$ & $428 \quad(9.3)$ & $346(23.7)$ & $153(19.3)$ & $61 \quad(22.9)$ & $82(24.9)$ \\
\hline $75-84$ & $119(16.5)$ & $245 \quad(5.4)$ & $135 \quad(9.3)$ & $183(23.1)$ & $56 \quad(21.1)$ & $62(18.8)$ \\
\hline$\geq 85$ & $45 \quad(6.2)$ & $88 \quad(1.9)$ & $28 \quad(1.9)$ & $101(12.7)$ & $18 \quad(6.8)$ & $25(7.6)$ \\
\hline
\end{tabular}

\begin{tabular}{|c|c|c|c|c|}
\hline \multirow[b]{2}{*}{ Characteristic } & \multicolumn{4}{|c|}{ Non-HPV-related cancers*; no. (\%) of patients } \\
\hline & $\begin{array}{c}\text { Lung } \\
n=39956\end{array}$ & $\begin{array}{l}\text { Endometrium } \\
n=8843\end{array}$ & $\begin{array}{c}\text { Ovary } \\
n=5496\end{array}$ & $\begin{array}{c}\text { Colon } \\
n=24145\end{array}$ \\
\hline \multicolumn{5}{|l|}{ Sex } \\
\hline Male & $24363(61.0)$ & - & - & $12469(51.6)$ \\
\hline Female & $15593(39.0)$ & $8843(100.0)$ & $5496(100.0)$ & $11676(48.4)$ \\
\hline \multicolumn{5}{|l|}{ Age, yr } \\
\hline$<35$ & $133 \quad(0.3)$ & $101 \quad(1.1)$ & $434 \quad(7.9)$ & $227 \quad(0.9)$ \\
\hline $35-44$ & $848 \quad(2.1)$ & $482 \quad(5.5)$ & $629(11.4)$ & $745 \quad(3.9)$ \\
\hline $45-54$ & $3939 \quad(9.9)$ & 1469 (16.6) & 1081 (19.7) & $2248 \quad(9.3)$ \\
\hline $55-64$ & $9563(23.9)$ & 2988 (33.8) & 1182 (21.5) & $4550(18.8)$ \\
\hline $65-74$ & $13558(33.9)$ & $2302 \quad(26.0)$ & $1128 \quad(20.5)$ & $7012(29.0)$ \\
\hline $75-84$ & $9720(24.3)$ & 1202 (13.6) & 764 (13.9) & $6665(27.6)$ \\
\hline$\geq 85$ & 2193 & $299 \quad(3.4)$ & $278 \quad(5.1)$ & $2698(11.2)$ \\
\hline
\end{tabular}


(annual percentage change $0.2, p=0.40$ ). The incidence of ovarian cancer was stable until 1994 (annual percentage change 0.4, $p=0.43$ ), after which it decreased (annual percentage change $-3.5, p=0.04$ ) (Appendix 1c). The age-standardized incidence of colon cancer among women consistently decreased, from 30 (95\% CI 29.5-30.5) per 100000 to 26 (95\% CI 25.5-26.5) per $100000(p<0.001)$. Among men, the incidence of colon cancer increased between 1975 and 1997 (annual percentage change 1.0, $p<0.001$ ) and was stable between 1997 and 2008 (annual percentage change $-0.8, p=0.54$ ) (Appendix 1d).

For numbers of cases by cancer type, 5-year period, age group and sex, see Appendix 2.

\section{Interpretation}

We observed an increase in the incidence of noncervical, HPV-related cancers in Alberta from 1975 to 2009, specifically cancers of the anus and oropharynx. Although not statis- tically significant, the greater increases in oropharyngeal cancer among younger men and in anal cancer among younger women warrant monitoring or further investigation to determine whether these trends continue or increase. There was little change in incidence over time for other HPV-associated cancers (vulvar, vaginal and penile). In comparison, the agestandardized incidence of cervical cancer declined over the study period, as anticipated because of established screening programs. The incidence of non-HPV-related cancers (lung, endometrial, ovarian and colon) decreased in the latter years of the study, except for lung cancer among women, which continued to increase, reflecting historic smoking patterns.

Similar trends in the incidence of HPV-associated cancers have been reported elsewhere in Canada and other regions in the world. Recent studies evaluating oropharyngeal cancer have suggested that, in contrast to head and neck cancers, which are associated with tobacco and alcohol use, the incidence of HPV-associated cancers is on the rise, ${ }^{10-14}$ although
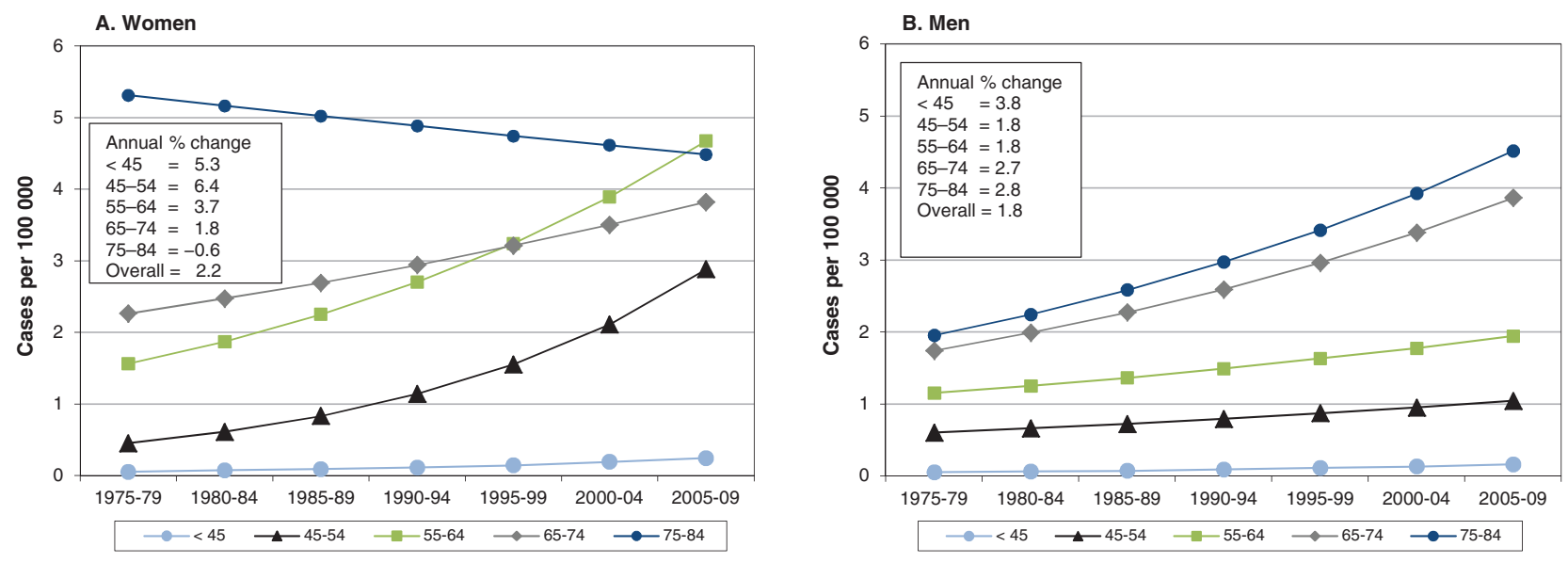

Figure 1: Age-specific trends in incidence of anal cancers in Alberta, Canada, 1975-2009, among women (A) and men (B).

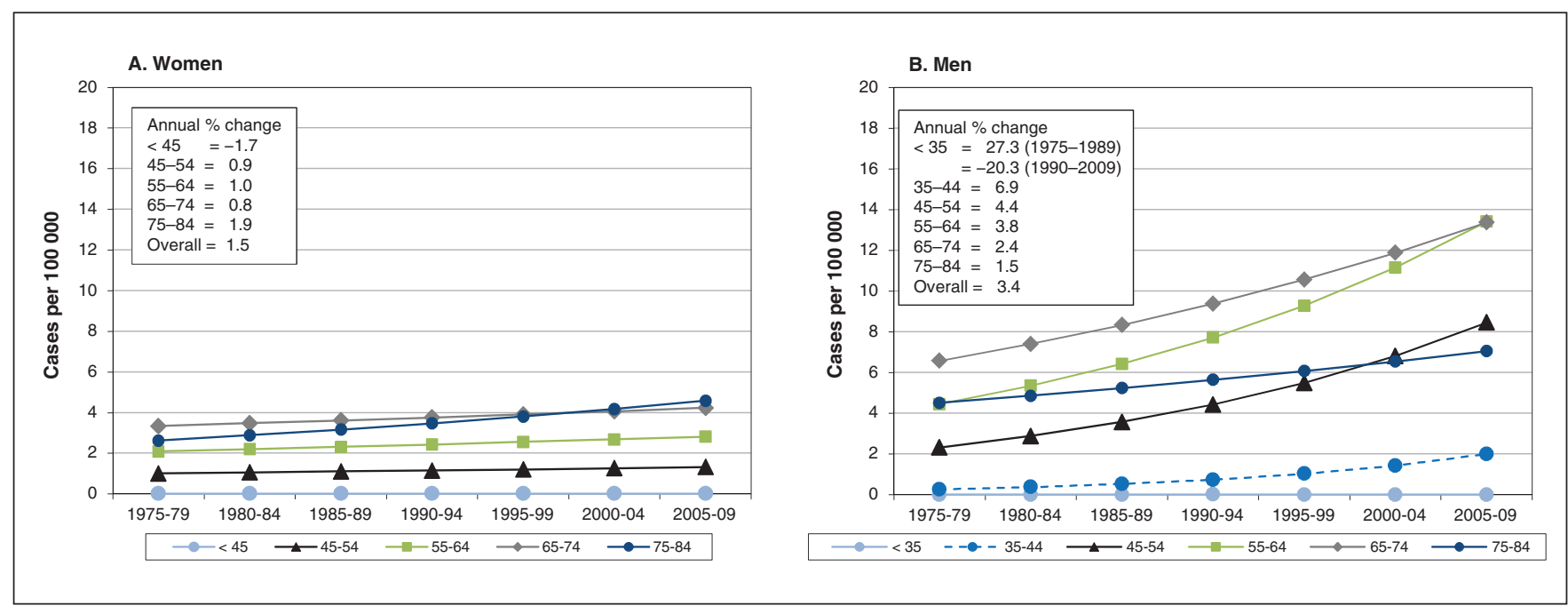

Figure 2: Age-specific trends in incidence of oropharyngeal cancers in Alberta, Canada, 1975-2009, among women (A) and men (B). 
incidence varies by country, ethnicity or race, and sex..$^{14,15}$ Data from the Canadian Cancer Registry show that the age-standardized incidence of HPV-associated oropharyngeal cancer increased from 1.6 per 100000 in 1992 to 2.6 per 100000 in 2009. ${ }^{16}$ A recent study from British Columbia mirrors the overall Canadian trends. ${ }^{17}$ We observed that the increase in incidence of oropharyngeal cancer among men was twice that among women (annual percentage change 3.4 v. 1.5) between 1975 and 2009. Similarly, both Canadian ${ }^{16}$ and $\mathrm{US}^{18}$ data show that the increased incidence of HPV-associated head and neck cancers was greater among men, most notably the younger groups. In 2 previously reported Canadian studies, ${ }^{16,17}$ ethnic minorities appeared to be at higher risk than the general population of both oropharyngeal cancer and oral cavity cancers. In the US, non-Hispanic white and Hispanic populations experienced greater increases in the incidence of HPV-associated head and neck cancers than other groups. ${ }^{18}$ It is unclear whether racial and ethnic variations reflect differences in oncogenic HPV infection or other risk factors. Because ethnicity was not consistently available in our registry data, we were unable to determine whether there were any potential differences in HPV-related cancer incidence by ethnic group in Alberta.

For anal cancer, studies from the US, ${ }^{19}$ Australia $^{20}$ and several European countries ${ }^{12,21-23}$ have reported an increase in incidence over time. As was also observed in our study, the incidence of anal cancer in most countries is higher among women than among men. Among the other HPV-related cancers we examined (vulvar, vaginal and penile), we noted a significant increase only in the incidence of vulvar cancer, but the change was small. We observed little change in the incidence over time of vaginal and penile cancers, although the rarity of these tumours limits the ability to distinguish small differences. Reports of increasing incidence of vulvar, vaginal and penile cancers have been observed in some parts of the world.

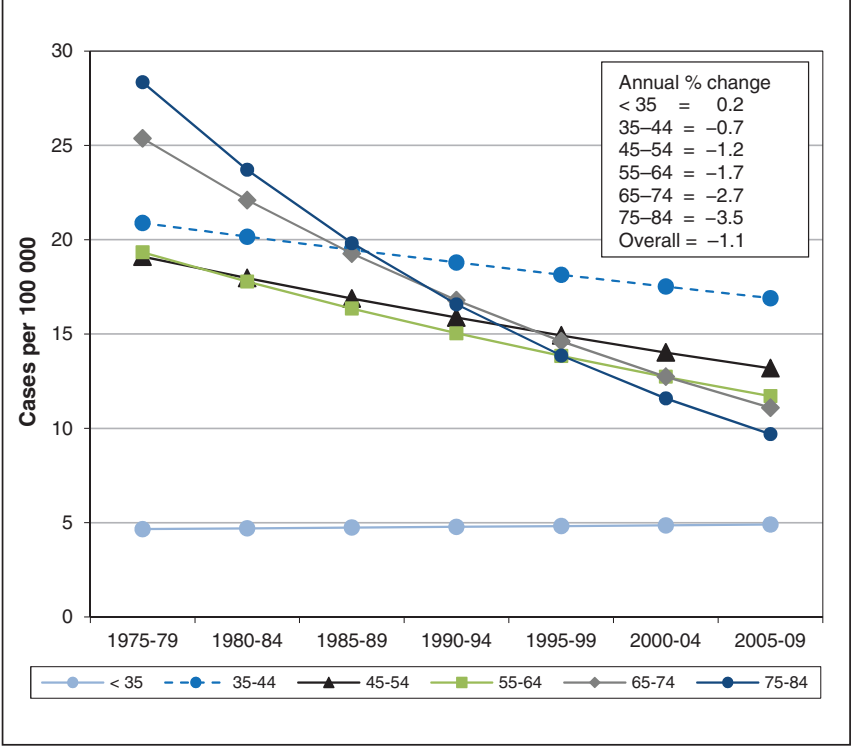

Figure 3: Age-specific trends in incidence of cervical cancers in Alberta, Canada, 1975-2009.
In both Denmark ${ }^{24}$ and the Netherlands,${ }^{25}$ the incidence of penile squamous cell carcinomas has been increasing. Similarly, in the US, the incidence of invasive vulvar carcinoma increased $1.0 \%$ per year between 1973 and 2004. ${ }^{26}$ As with other HPV-associated cancers, the rates of invasive vaginal, vulvar, penile and anal carcinomas have all been reported to vary by race and ethnicity. ${ }^{27-29}$

\section{Strengths and limitations}

Strengths of this study include the evaluation of all known $\mathrm{HPV}$-associated cancers in a comparative analysis of data from a single centralized cancer registry over 4 decades. The quality of the Alberta Cancer Registry data is known to be high but may be affected by incorrect or incomplete data or changes in identification because of the use of new diagnostic techniques over time. In addition, data were collected retrospectively from the registry without corroborating chart reviews. Therefore, the potential for miscoding remains, as has been described in relation to registry data.

A weakness of this study is the inability to confirm histologically the definitive HPV status in tumours. Although we included tumour sites with known HPV-association, we may have over- or underestimated the incidence of true HPVassociated cancers.

Other important risk factors, such as smoking, may influence the incidence of various cancers. However, the decreasing incidence of most tobacco-related cancers over the same study period suggests that changes related to smoking are unlikely to account for the rising trends we observed in cancers of the anus and oropharynx. Past behavioural changes, which may be influenced by demographic fluctuations, may also influence exposure to risk factors and the current incidence of various cancers. Our study was not aimed at assessing behavioural changes, nor did we attempt to explore potential underlying causes of the observed rise in incidence of HPV-associated cancers. Certainly, the observation that HPV-associated cancers are on the rise raises the need for more study of behavioural changes that may underlie these trends.

\section{Conclusion}

Our findings showed an increase in the incidence of noncervical, HPV-related cancers in Alberta from 1975 to 2009, specifically cancers of the oropharynx and anus. This increase has occurred at a time when cervical cancer incidence in the province has been on the decline, likely in relation to widespread implementation of screening programs. The increases in the incidence of oropharyngeal cancer among younger men and of anal cancer among younger women, although not significant, are disturbing, because there are no screening programs for early detection of either of these cancers. To have a large impact on the prevention of these HPV-associated cancers, vaccination programs should be considered for males as well as females. Both oropharyngeal cancer and anal cancers are associated with substantial morbidity, and their treatment comes at a cost of substantial toxicity; therefore, education and prevention programs, such as the HPV vaccination program launched in Alberta and elsewhere, ${ }^{30}$ are urgently required. 


\section{References}

1. Parkin DM. The global health burden of infection-associated cancers in the year 2002. Int 7 Cancer 2006;118:3030-44.

2. Parkin DM, Bray F. Chapter 2: the burden of HPV-related cancers. Vaccine 2006;24:(Suppl 3)S3/11-25.

3. IARC Working Group on the Evaluation of Carcinogenic Risks to Humans. Human papillomaviruses. IARC Monogr Eval Carcinog Risks Hum 2007;90:1-636. Available: http://monographs.iarc.fr/ENG/Monographs/vol90/index.php (accessed 2013 Jun. 20).

4. Hatcher J, editor. Cervical cancer: 2010 report on cancer statistics in Alberta. Edmonton (AB): Cancer Care, Alberta Health Services; 2012. Available: www.albertahealthservices.ca/poph/hi-poph-surv-cancer-cervical-2010.pdf (accessed accessed 2013 Jun. 20).

5. Chaturvedi AK. Beyond cervical cancer: burden of other HPV-related cancers among men and women. 7 Adolesc Health 2010;46(4 Suppl):S20-6.

6. Robinson D, Coupland V, Møller H. An analysis of temporal and generational trends in the incidence of anal and other HPV-related cancers in Southeast England. Br 7 Cancer 2009;100:527-31.

7. Eggertson L. Provinces weighing HPV vaccination of boys. CMAf 2012;184: E250-1.

8. Alberta Cancer Registry. 2009: annual report of cancer statistics. Edmonton (AB): Cancer Care, Alberta Health Services; 2012.

9. Kim HJ, Fay MP, Feuer EJ, et al. Permutation tests for joinpoint regression with applications to cancer rates [published erratum in 2001;20:655]. Stat Med 2000; 19: 335-51.

10. Blomberg M., Nielsen A., Munk C., et al. Trends in head and neck cancer incidence in Denmark, 1978-2007: focus on human papillomavirus associated sites. Int 7 Cancer 2011;129:733-41.

11. Hocking JS, Stein A, Conway EL, et al. Head and neck cancer in Australia between 1982 and 2005 show increasing incidence of potentially HPV-associated oropharyngeal cancers. Br 7 Cancer 2011;104:886-91.

12. Ryerson AB, Peters ES, Coughlin SS, et al. Burden of potentially human papillomavirus-associated cancers of the oropharynx and oral cavity in the US, 1998-2003. Cancer 2008;113:2901-9.

13. Chaturvedi AK, Engels EA, Anderson WF, et al. Incidence trends for human papillomavirus-related and -unrelated oral squamous cell carcinomas in the United States. 7 Clin Oncol 2008;26:612-9.

14. Herrero R, Castellsagué X, Pawlita M, et al. Human papillomavirus and oral cancer: the International Agency for Research on Cancer multicenter study. 7 Natl Cancer Inst 2003;95:1772-83.

15. Gillison ML, D'Souza G, Westra W, et al. Distinct risk factor profiles for human papillomavirus type 16-positive and human papillomavirus type 16-negative head and neck cancers. 7 Natl Cancer Inst 2008;100:407-20.

16. Forte T, Niu J, Lockwood G, et al. Incidence trends in head and neck cancers and human papillomavirus (HPV)-associated oropharyngeal cancer in Canada, 1992-2009. Cancer Causes Control 2012;23:1343-8.

17. Auluck A, Hislop G, Bajdik C, et al. Trends in oropharyngeal and oral cavity cancer incidence of human papillomavirus (HPV)-related and HPV-unrelated sites in a multicultural population: the British Columbia experience. Cancer 2010;116:2635-44.

18. Cole L, Polfus L, Peters ES. Examining the incidence of human papillomavirus-associated head and neck cancers by race and ethnicity in the U.S., 1995-2005. PLoS One 2012;7:e32657.

19. Goodman MT, Hernandez BY, Shvetsov YB. Demographic and pathologic differences in the incidence of invasive penile cancer in the United States, 1995-2003. Cancer Epidemiol Biomarkers Prev 2007;16:1833-9.

20. Jin F, Stein AN, Conway EL, et al. Trends in anal canal cancer in Australia, 1982-2005. Vaccine 2011;29:2322-7.

21. Nielsen A, Munk C, Kjaer SK. Trends in incidence of anal cancer and high-grade anal intraepithelial neoplasia in Denmark, 1978-2008. Int 7 Cancer 2012; 130:1168-73.

22. Goldman S, Glimelius B, Nilsson B, et al. Incidence of anal epidermoid carcinoma in Sweden 1970-1984. Acta Chir Scand 1989;155:191-7.

23. Brewster DH, Bhatti LA. Increasing incidence of squamous cell carcinoma of the anus in Scotland, 1975-2002. Br 7 Cancer 2006;95:87-90.

24. Baldur-Felskov B, Hannibal CG, Munk C, et al. Increased incidence of penile cancer and high-grade penile intraepithelial neoplasia in Denmark 1978-2008: a nationwide population-based study. Cancer Causes Control 2012;23:273-80.

25. Graafland NM, Verhoeven RH, Coebergh JW, et al. Incidence trends and survival of penile squamous cell carcinoma in the Netherlands. Int 7 Cancer 2011; 128:426-32.

26. Bodelon C, Madeleine MM, Voigt LF, et al. Is the incidence of invasive vulvar cancer increasing in the United States? Cancer Causes Control 2009;20:1779-82.

27. Wu X, Matanoski G, Chen VW, et al. Descriptive epidemiology of vaginal cancer incidence and survival by race, ethnicity, and age in the United States. Cancer 2008;113(10 Suppl):2873-82.

28. Watson M, Saraiya M, Wu X. Update of HPV-associated female genital cancers in the United States, 1999-2004. F Womens Health (Larchmt) 2009;18:1731-8.

29. Baandrup L, Varbo A, Munk C, et al. In situ and invasive squamous cell carcinoma of the vulva in Denmark 1978-2007: a nationwide population-based study. Gynecol Oncol 2011;122:45-9.

30. National Advisory Committee on Immunization. Update on human papillomavirus (HPV) vaccines. An Advisory Committee Statement. Can Commun Dis Rep 2012: 38:1-62.

Affiliations: Surveillance and Reporting (Shack), Cancer Control Alberta, Alberta Health Services; Department of Oncology (Shack, Lau, Doll, Hao), Community Health Sciences (Shack), Faculty of Medicine, University of Calgary; Division of Radiation Oncology (Lau, Doll), Department of Oncology, Tom Baker Cancer Centre, Alberta Health Services; Department of Mathematics and Statistics (Huang), University of Calgary; Division of Medical Oncology (Hao), Department of Oncology, Tom Baker Cancer Centre, Alberta Health Services, Calgary, Alta.

Contributors: Lorraine Shack, Harold Lau, Longlong Huang, Corinne Doll and Desirée Hao each contributed to the concept and study design, drafted and revised the paper and approved the final version submitted for publication. All of the authors agree to act as guarantors for this work.

Funding: Funded by Joe's Team Triathlon/Alberta Cancer Foundation (ref. 26133). The study sponsors had no role in the design of the study, the collection, analysis or interpretation of data, the writing of the report or the decision to submit the article for publication.

Supplemental information: For reviewer comments and the original submission of this manuscript, please see www.cmajopen.ca/content/2/3 /E127/suppl/DC1 